Research Paper

\title{
Schedulability Comparisons between Priority Queue and FIFO Queue for CAN Messages with Offsets
}

\author{
Yang Chen ${ }^{1)}$ Ryo Kurachi ${ }^{2)}$ Gang Zeng ${ }^{3)}$ Hiroaki Takada ${ }^{4)}$ \\ 1). 2). 4) Center for Embedded Computing Systems, Graduate School of Information Science, Nagoya University \\ Furo-cho, Chikusa-ku, Nagoya, 464-8603, Japan (E-mail: \{chenyang, kurachi, hiro\}@ertl.jp) \\ 3) Graduate School of Engineering, Nagoya University \\ Furo-cho, Chikusa-ku, Nagoya, 464-8603, Japan (E-mail: sogo@ertl.jp)
}

Received on August 24, 2012

\begin{abstract}
The Controller Area Network (CAN) is a widely used in-vehicle network and its schedulability is particularly important. In a CAN system, implementing the priority queues is better than FIFO queues with respect to the schedulability, which is widely recognized by users. In this paper, we investigate whether and how much implementing the priority queues is better than FIFO queues when the offset assignment for CAN messages is conducted. Our results show that usually the priority queues are better, whereas the FIFO queues are better in some specific situations. Furthermore, we propose a new scheduling method for obtaining the advantages of both queues, which improves the schedulability effectively.
\end{abstract}

KEY WORDS: information, communication, and control, controller area network, inter-vehicle communication, real-time systems, real-time communications, scheduling theory, schedulability analysis [E2]

\section{Introduction}

The Controller Area Network (CAN) was designed as a simple, efficient and robust broadcast communications bus for invehicle networks; and it has been widely used by automotive manufacturers in recent decades. In the utilization of CAN, realtime constraint is particularly important, which requires that all the messages should meet their deadlines. For this reason, schedulability analysis for CAN messages has been proposed ${ }^{(2)(3)}$, which calculate the worst-case response time (WCRT) of every message, and compare it with the deadline to determine whether the real-time constraint is guaranteed.

However, the sophistication and complexity of in-vehicle communications have seen a notable increase in the number of transmitted messages, and make the bus utilization higher and higher in recent years. In order to match the real-time constraint in this situation, many researches investigated the scheduling for CAN messages. Effective approaches such as message queue selection, offset assignment have been proposed ${ }^{(4)(5)(6)(7)(8)}$.

Priority queue and FIFO queue are the two common queues almost supported by all CAN controllers. Although using a FIFO queue obtains the simplicity and the faster queue management, which is seemed an attractive solution to improve the performance of the system ${ }^{(4)}$, the disadvantage of the FIFO queue is that it introduces significant priority inversion - the situation that a low-priority message delays high-priority messages — and results in degraded schedulability of CAN messages. For this reason, it is better to implement a priority queue than a FIFO queue for a CAN device, which is recommended by previous work $^{(5)}$
The offset assignment for CAN messages is an effective approach for decreasing the WCRT, which has been confirmed on both the priority queues implemented systems and the FIFO queues implemented systems ${ }^{(9)(12)}$. However, the issue that whether the priority queue is still better than the FIFO queue when the offset assignment is conducted has not been concerned by previous work.

In this paper, we conduct the experiments based on the WCRT calculation methods of references (9) and (12) to compare the schedulability between the priority queue and FIFO queue for CAN messages with offsets. In the experiments, a large number of simulated message sets generated with the bus utilization from $30 \%$ to $80 \%$, and a real-world messages set provided by an automobile manufacturer are investigated. Based on the comparison results, first, we summarize the pros and cons of both the queues; and show that the FIFO queue is better in some specific situations. Second, we introduce a new scheduling method that uses the two types of queues in one station for obtaining the advantages of both schemes. The experimental results on a real-world message set show that the combination of the priority and FIFO queues can achieve higher schedulability than either a priority or a FIFO queue alone.

The rest of this paper is organized as follows. Section 2 describes the terminology, notation and system models. Section 3 introduces the related work. Section 4 shows the schedulability comparisons, followed by Section 5 that discusses the differences between the priority queue and FIFO queue when the worst case occurs, and summarizes the pros and cons. Section 6 proposes the new scheduling method that uses the two types of queues in one station, and shows the evaluations. The paper is concluded in Section 7. 


\section{System Model}

\subsection{The Controller Area Network}

CAN is a serial broadcast bus that sends and receives short real-time control messages ${ }^{(1)}$. The CAN bus is designed to connect several stations and to operate at a maximum speed of 1 $\mathrm{Mbit} / \mathrm{sec}$. A simple CAN bus architecture is shown in Fig. 1.

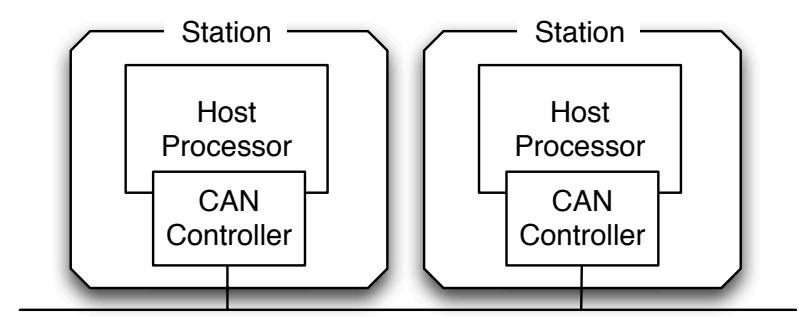

CAN BUS

Fig. 1 An example of CAN bus architecture

Each message transmitted on a CAN bus is required to have a unique identifier (ID). The ID may be 11 bits (standard format) or 29 bits (extended format). When a CAN controller attempts to transmit a message, it has to wait until a bus idle period is detected. If two or more stations start to transmit messages simultaneously, the bus arbitration is triggered and the ID is used as a priority (a message with a smaller ID has higher priority) to determine which message will be transmitted among those contending for the bus.

The messages are queued in the stations before being transmitted to CAN bus. The queue is memory, implemented as dual ports and shared between the host processor and CAN controller. According to the ordering mechanism, the queues are classified to priority and FIFO. Fig. 2 is an example of how the queues work. In this example, the host processor queues ID:1 message into the queue in which ID:2 and ID:3 messages already existed. In Fig. 2(a) where a priority queue is implemented, the

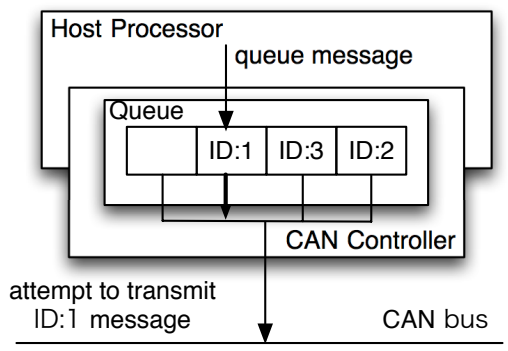

(a) A priority queue equipped CAN station

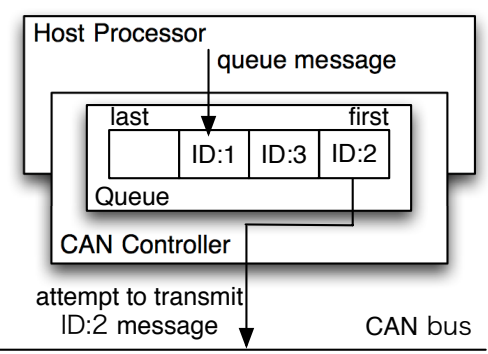

(b) A FIFO queue equipped CAN station

Fig. 2 Message queueing
CAN controller attempts to transmit the highest priority message (ID:1) to CAN bus first. On the other side, when a FIFO queue is implemented, as shown in Fig. 2(b), the CAN controller attempts to transmit the oldest message (ID:2) to the CAN bus first.

\subsection{Notations}

The system is assumed to be composed of a single CAN bus and several CAN stations. The stations, denoted $U_{I}\left(I \in Z^{+}\right)$, are classified according to the type of their queues. Therefore, a PQstation implements a priority queue, whereas a FQ-station implements a FIFO queue. A PQ-station is assumed to provide that the highest priority message in the station is entered into arbitration whenever the bus arbitration starts. A FQ-station is assumed to ensure that the oldest message in the station is entered into arbitration whenever the bus arbitration starts.

The transmitted messages in a system are considered as a message set. Each message, denoted $\tau_{i}\left(i \in Z^{+}\right)$, has static properties which comprise priority $P_{i}$, maximum transmission time $C_{i}$, period $T_{i}$, and offset $O_{i}$.

The value of $P_{i}$ is equal to the ID of $\tau_{i} . \tau_{i}$ has higher priority than $\tau_{j}$ if $P_{i}<P_{j}$. Since each CAN message has a unique ID in the system, for any two messages $\tau_{i}, \tau_{j}(i \neq j), P_{i} \neq P_{j}$ holds.

The maximum transmission time $C_{i}$ is determined by the number of data bytes, bit stuffs and the inter-frame space, which is calculated by the formula below ${ }^{(11)}$.

$$
C_{i}=\left(\left\lfloor\frac{g+8 s_{i}-1}{4}\right\rfloor+g+8 s_{i}+13\right) t_{b i t}
$$

In the formula $g=34$ for standard CAN (11-bit IDs) or $g=54$ for extended CAN (29-bit IDs). $t_{b i t}$ denotes the time it takes to transmit one bit. $s_{i}$ denotes the number of bytes in the data field of the message.

It is assumed that all messages are periodic messages and reoccur infinitely. Therefore, $T_{i}$ is the interval between two consecutive occurrence of $\tau_{i}$; and $O_{i}$ is the instant at which $\tau_{i}$ firstly occurs. Each instant that $\tau_{i}$ occurs, denoted $A_{i}^{n}\left(\mathrm{n}=Z_{0}^{+}\right)$, is defined as an arrival of $\tau_{i}$. Hence it is $A_{i}^{0}=O_{i}$.

The properties of a message $\tau_{i}$ are described in Fig. 3. In the figure, the horizontal axis represents time; up-pointing arrows represent the arrivals. The white boxes represent messages transmissions; and the number in each box represents the priority.

The WCRT of $\tau_{i}$, denoted $R_{i}$, is the worst-case delay that $\tau_{i}$ may experience between its arrival and transmission completion. $R_{i}$ is compared with the deadline, denoted $D_{i}$, to determine whether $\tau_{i}$ is schedulable or not $-\tau_{i}$ is schedulable if $R_{i} \leq D_{i}$. Therefore, the system is schedulable if $R_{i} \leq D_{i}$ holds for all the messages.

Because it is hard to evaluate the schedulability of same system scheduled by different method, deadline rate and worstcase response time rate (WCRR) have been proposed for the

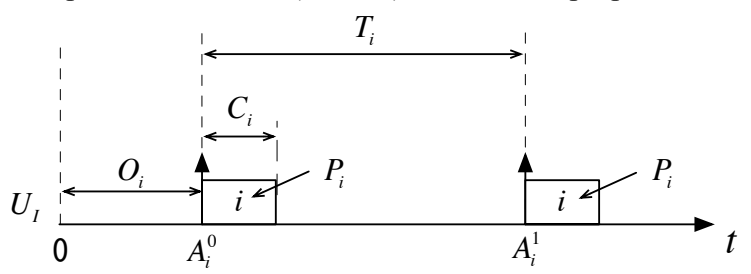

Fig. 3 The properties of a message 
quantitative evaluation of schedulability ${ }^{(8)(10)(12)}$. Considering the fact that a deadline is always assigned according to the ratio of the period in a real automotive system, the deadline rate of a message is defined as the ratio of deadline to the period, and the WCRR is the ratio of WCRT to the period. Let $D R_{i}$ and $W C R R_{i}$ denote the deadline rate and WCRR of $\tau_{i}$ respectively. $D R_{i}$ and $W C R R_{i}$ are calculated by following equations:

$$
\begin{aligned}
& D R_{i}=\left(D_{i} / T_{i}\right) * 100 \% \\
& W_{C R R_{i}}=\left(R_{i} / T_{i}\right) * 100 \%
\end{aligned}
$$

Based on these definitions, $\tau_{i}$ is schedulable if $W C R R_{i} \leq D R_{i}$. Furthermore, for the same system scheduled by different methods $\mathrm{A}$ and $\mathrm{B}$, the schedulability of method $\mathrm{A}$ is higher than $\mathrm{B}$ if the maximum $W C R R_{i}$ of $\mathrm{A}$ is smaller than that of $\mathrm{B}$.

\subsection{Effects of the Offset Assignment}

The offset assignment decreases the WCRT by avoiding multiple messages being transmitted simultaneously. A comparison of messages transmission with and without offsets is shown in Fig. 4. In the example, $U_{l}$ has two messages $\tau_{l}\left(P_{l}=1\right.$, $\left.C_{l}=1, T_{1}=4\right)$ and $\tau_{2}\left(P_{2}=2, C_{2}=1, T_{2}=8\right) ; U_{2}$ has one message $\tau_{3}\left(P_{3}=3, C_{3}=1, T_{3}=16\right)$. In the situation where no offset is assigned (i.e., offsets of all messages are 0 ), $\tau_{1}$ delays $\tau_{2}$; $\tau_{1}$ and $\tau_{2}$ delay $\tau_{3}$ in the worst case. While assigning an offset value 2 to $O_{2}$ avoids $\tau_{1}$ and $\tau_{2}$ to be transmitted simultaneously, hence the WCRTs of both $\tau_{2}$ and $\tau_{3}$ are decreased.

\section{Related Work}

Schedulability analysis for CAN messages has been firstly investigated by Tindell et $\mathrm{al}^{(2)(3)}$. They proposed the WCRT

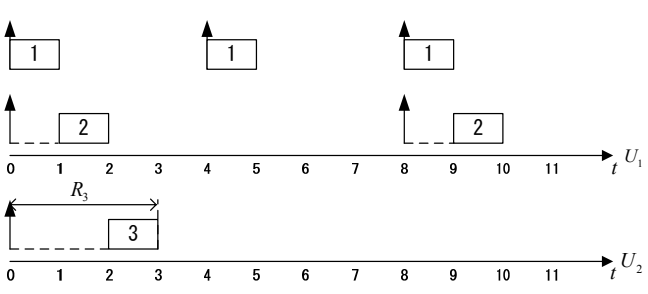

(a) Without offset

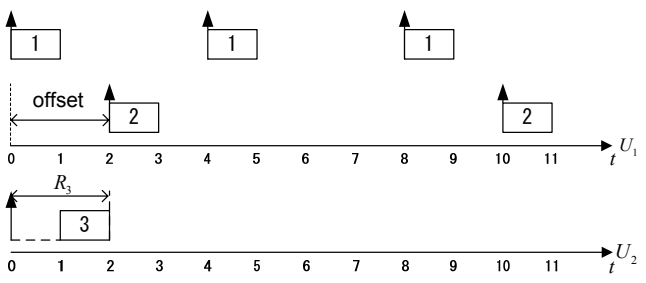

(b) With offset

Fig. 4 Effects of the offset assignment

calculating method so that the real-time constraint of CAN message can be guaranteed. This research has then been improved by successive studies. The most recent achievement is the study proposed by Davis et al, which corrected some mistakes and revised the original analysis method ${ }^{(11)}$.

According to these studies, it is known that a CAN message will be delayed by all the other high-priority messages in the worst case. To relieve this problem, the offset assignment for CAN messages has been discussed by Matustani et $\mathrm{al}^{(6)}$, Grenier et $\mathrm{al}^{(7)}$ and Chen et $\mathrm{al}^{(8)}$, respectively. Meanwhile, Iiyama et al and Kurachi et al proposed the WCRT analysis methods for offsetassigned CAN messages, which have confirmed that the offset assignment reduces the WCRT effectively ${ }^{(9)(10)}$.

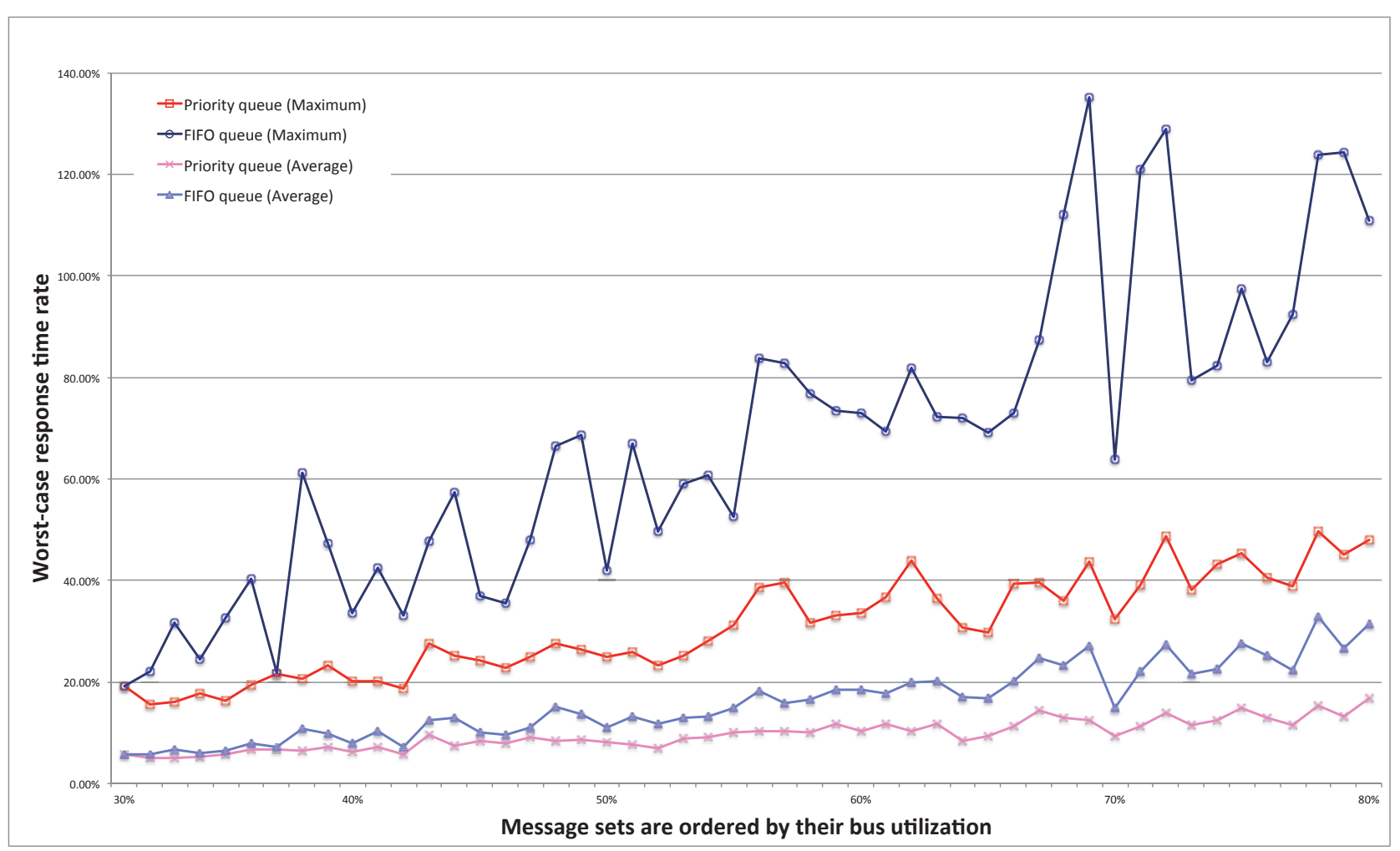

Fig. 5 The WCRR comparing results on 50 message sets which are generated by the NETCARBENCH with bus utilization from $30 \% \sim 80 \%$ 
Because all the above researches aimed at the systems that only comprise PQ-stations, Davis et al proposed the analysis method which is able to calculate the WCRTs of messages in the FQ-stations used systems ${ }^{(5)}$. The study illustrates that implementing PQ-stations is better than FQ-stations with respect to the schedulability.

While Davis's study did not consider the offset assignment. For this reason, Chen et al proposed their method for calculating the WCRT of the FIFO-queued messages with offsets. The study has confirmed that the offset assignment also effectively decreases the WCRT for the FQ-stations implemented systems ${ }^{(12)}$.

However, to the best of our knowledge, no published research has compared the two types of queues in a CAN system, with the respect to the WCRT, when the offset assignment is conducted. This leads our studies in this paper.

\section{Schedulability Comparison Experiments and Results}

In this section we explain the schedulability comparison experiments and their results. The experiments are conducted on 50 test systems. All the stations in each system are assumed to be PQ-stations when the schedulability results corresponding to the priority queue are calculated, whereas the schedulability results corresponding to the FIFO queue are calculated by assuming all the stations to be FQ-stations. Iiyama's method ${ }^{(9)}$ and Chen's method $^{(12)}$ are employed in the WCRT calculation for PQ-stations implemented system and FQ-stations implemented system, respectively.

The message sets of the test systems are generated by the NETCARBENCH ${ }^{(13)}$ - a very popular automotive message sets generator. The following configurations are conducted on the message sets:

(1) The message sets are generated with the bus utilization from $30 \%$ to $80 \%$.

(2) Offset assignment for each message set is conducted by the NETCARBENCH.

(3) Deadline of each message is assigned to equal to its period. Hence deadline rate of each message is $100 \%$.

(4) Priorities of the messages are assigned based on the deadline monotonic rule - the message with shorter deadline gets higher priority.

The results are depicted in Fig. 5 and Fig. 6. In the figures, the red lines with square markers depict the maximum WCRR or WCRT results when PQ-stations are implemented; the dark blue lines with cycle markers depict the maximum WCRR or WCRT results when FQ-stations are implemented; the pink lines with cross markers depict the average WCRR or WCRT results when PQ-stations are implemented; the light blue lines with triangle markers depict the average WCRR or WCRT results when FQstations are implemented.

First, we focus on the WCRR results shown in Fig. 5. We observe that the implementation of PQ-stations awards higher schedulability in all the tested systems - its maximum WCRR of each message set is smaller. Moreover, all the messages meet their deadlines even the bus utilization is $80 \%$. On the other side, the implementation of FQ-stations causes both the average and maximum WCRRs to be increased. The increases are more obvious when the bus utilization becomes high. In the extreme situation, implementing FQ-stations causes the maximum WCRR to be over 3 times as large as that of PQ-stations used situation. However, we also observe that the differences in the maximum WCRR results are much larger than that in the average WCRR

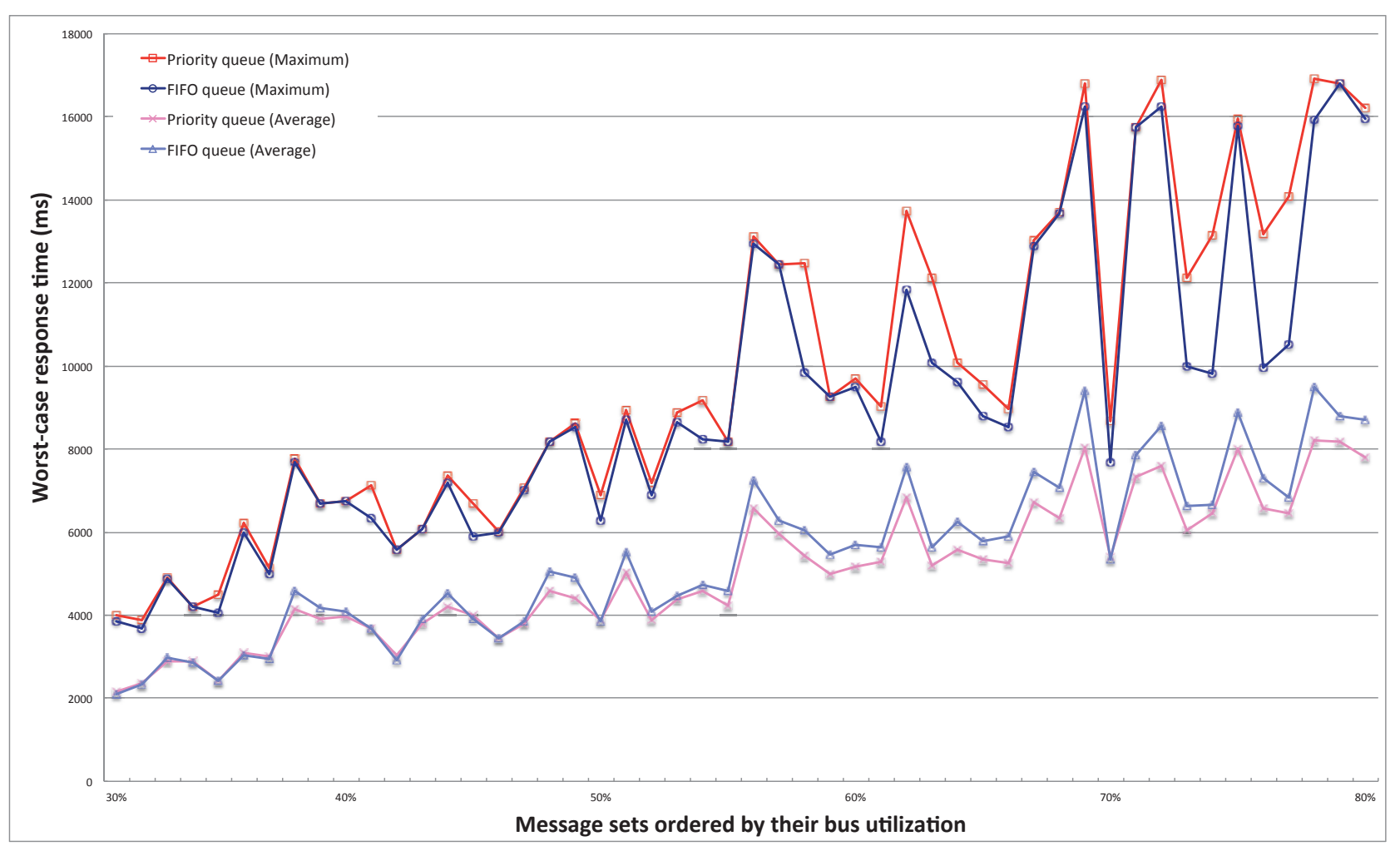

Fig. 6 The WCRT comparing results on 50 message sets which are generated by the NETCARBENCH with bus utilization from $30 \% \sim 80 \%$ 


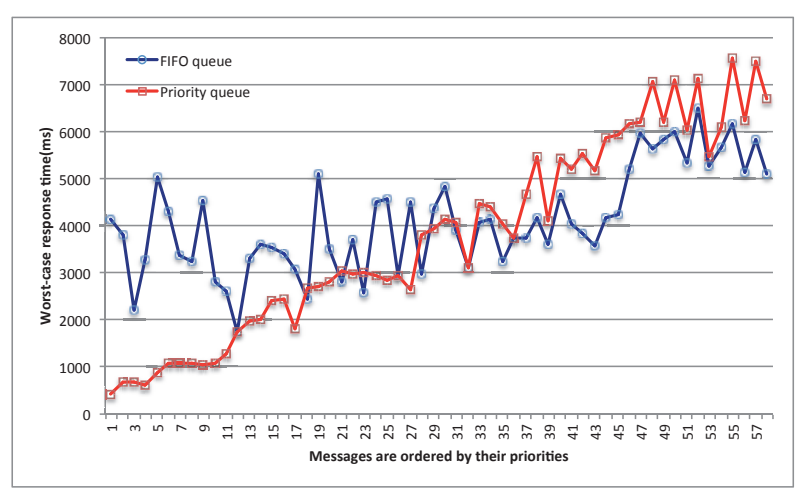

(a) The WCRT results

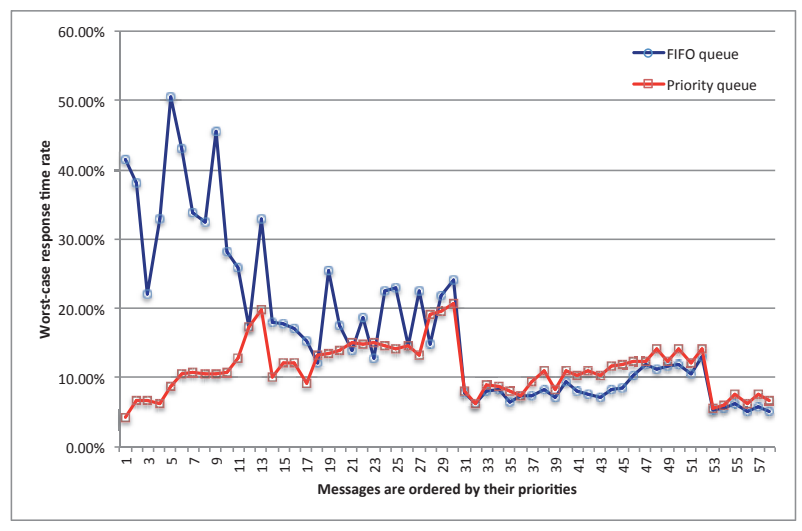

(b) The WCRR results

Fig. 7 The WCRRs and WCRTs of all messages in a message set with bus utilization $51.4 \%$

results, which indicates that the WCRRs of most messages do not become very worse when FQ-stations are used.

Second, we focus on the WCRT results shown in Fig. 6. We observe that although the implementation of FQ-stations still causes the increase of the average WCRTs, the maximum WCRTs of the FQ-stations implemented situations are smaller than that of the PQ-stations implemented situations. Also, the differences are more obvious with the increase of the bus utilization.

To show more detailed results, we pick out a message set from the above experiments and show the WCRRs and WCRTs of all messages in the message set. From the WCRT results, as shown in Fig. 7(a), we observe that with the decrease of the priorities (increase of the priority values) the WCRTs of messages in PQ-stations increase faster. The higher priority messages in PQ-stations have shorter WCRTs, whereas the lower priority messages in FQ-stations have shorter WCRTs. Moreover, when FQ-stations are implemented the maximum WCRT is shorter. However, the advantages of FQ-stations are not shown in the WCRR results. As shown in Fig. 7(b), the maximum WCRR is smaller when PQ-stations are implemented.

\section{Discussions}

Although Section 4 illustrated that in the tested systems implementing PQ-stations is better than FQ-stations with respect to the schedulability, the phenomenon - the maximum WCRT becomes short when the FQ-stations are implemented - indicates that the FQ-stations may be better in some specific situations. For this reason, in this section we discuss the differences between a PQ-station and FQ-station when the worst case occurs. Then, we summarize the pros and cons of both stations, and point out the specific situations where implementing FQ-stations are better with respect to the schedulability.

5.1. The Differences between a PQ-station and a FQ-station when the Worst Case Occurs

In a CAN system where all stations are PQ-stations and the offset assignment is conducted, the worst case of the message $\tau_{i}$ in the system is defined to be included in the following situations:

At least one message in each station with a priority higher than or equal to $P_{i}$ arrives simultaneously ${ }^{(9)}$.

All the situations that meet above descriptions must be treated as the candidates and be checked by the calculation in order to find the WCRT of $\tau_{i}$

When FQ-stations are implemented, the priority inversion causes that the messages with priorities lower than $\tau_{i}$ have also to be considered in the worst case. Hence the worst-case candidates of $\tau_{i}$ change to the follows:

At least one message in each station with a priority higher than or equal to $\left\{P_{j}\right\}$ arrives simultaneously. $\left\{P_{j}\right\}$ is a set of priorities of messages in the station that transmits $\tau_{i}^{(12)}$.

We give examples to explain the differences when the worst case occurs. Assume that a system consists of 3 stations and 6 messages. Properties of this message set are described in Table 1. When the system implements PQ-stations, one worst-case candidate of $\tau_{2}$ exists, in which $\tau_{2}$ arrives with $\tau_{1}$ simultaneously, as shown in Fig. 8(a). In the figure, $\tau_{6}$ exists because the message transmission on CAN bus is based on non-pre-emptive scheduling. Therefore, in the WCRT calculation, the low-priority message with the maximum transmission time is assumed to occupy the bus just before the simultaneously started instant in each candidate $^{(11)}$. Hence $R_{2}$ is 3 .

Table 1 An example message set

\begin{tabular}{|c|c|c|c|c|c|}
\hline$U_{I}$ & $\tau_{i}$ & $P_{i}$ & $T_{i}$ & $C_{i}$ & $O_{i}$ \\
\hline$U_{I}$ & $\tau_{6}$ & 6 & 8 & 1 & 0 \\
\hline$U_{2}$ & $\tau_{4}$ & 4 & 8 & 1 & 0 \\
$U_{2}$ & $\tau_{1}$ & 1 & 8 & 1 & 3 \\
$U_{2}$ & $\tau_{3}$ & 3 & 8 & 1 & 4 \\
\hline$U_{3}$ & $\tau_{2}$ & 2 & 8 & 1 & 1 \\
$U_{3}$ & $\tau_{5}$ & 5 & 8 & 1 & 0 \\
\hline
\end{tabular}

Because $U_{3}$ has two messages $\tau_{2}$ and $\tau_{5}$, the set of priorities of messages in $U_{3}$ is $\left\{P_{2}, P_{5}\right\}$. Therefore, when FQ-stations are implemented, there are 4 worst-case candidates of $\tau_{2}-(1) \tau_{4}$ and $\tau_{5}$; (2) $\tau_{1}$ and $\tau_{5}$; (3) $\tau_{3}$ and $\tau_{5}$; (4) $\tau_{1}$ and $\tau_{2}$ arrive simultaneously, respectively. The worst case is the candidate (2) $-\tau_{1}$ and $\tau_{5}$ arrive simultaneously —- as shown in Fig. 8(b). Hence $R_{2}$ is 5 . We observe that in the worst case, priority inversion in the station causes $\tau_{2}$ to wait for the transmission of $\tau_{5}$. Moreover, when $\tau_{5}$ attempts to be transmitted to the bus it has to wait for the transmission of $\tau_{3}$, hence $\tau_{2}$ is indirectly delayed by $\tau_{3}$.

Although the priority inversion causes the high-priority message $\tau_{2}$ to experience longer WCRT, the WCRT of a low- 


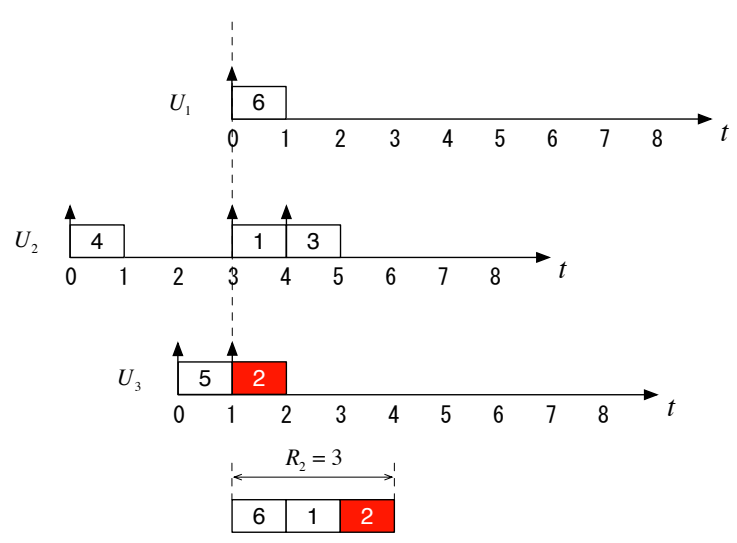

(a) The worst case of message $\tau_{2}$ in a priority queue

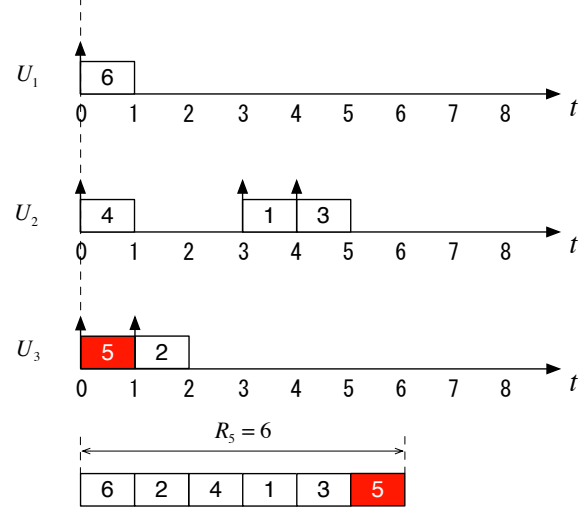

(c) The worst case of message $\tau_{5}$ in a priority queue

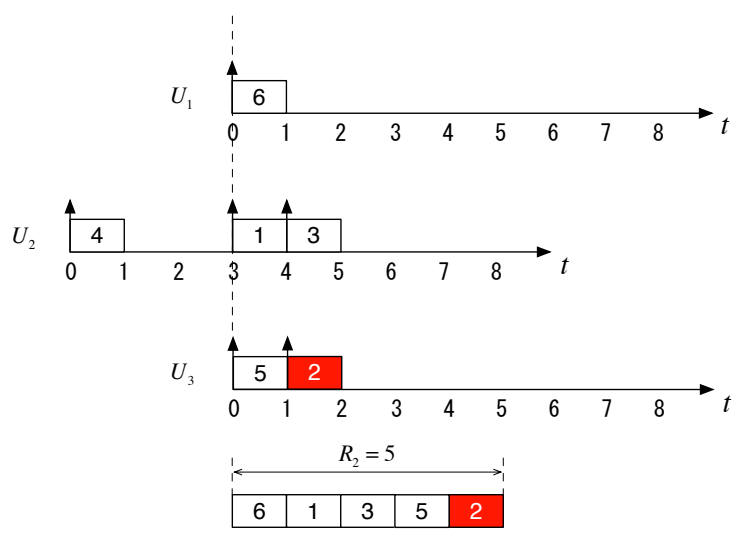

(b) The worst case of message $\tau_{2}$ in a FIFO queue

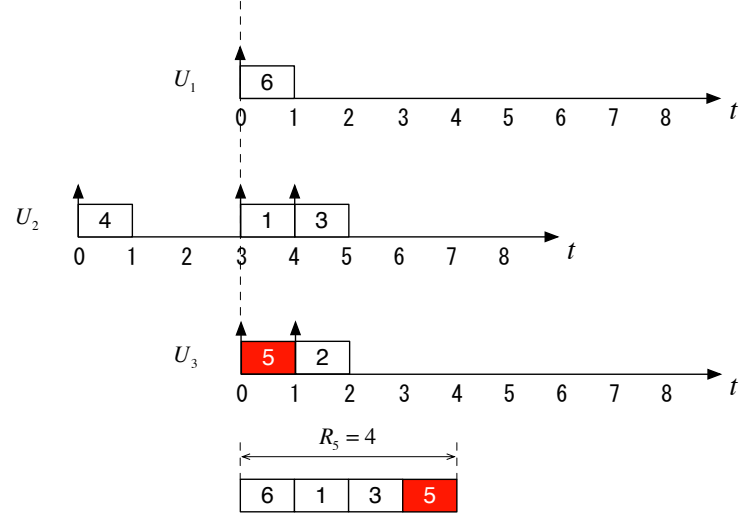

(d) The worst case of message $\tau_{5}$ in a FIFO queue

Fig. 8 The differences between a PQ-station and a FQ-station when the worst case occurs

priority message - for example, $\tau_{5}$ - can be decreased by it. The worst cases of $\tau_{5}$ is shown in Fig. 8(c)(d). When the system implements PQ-stations, the worst case of $\tau_{5}$ occurs when $\tau_{5}$ and $\tau_{1}$ arrive simultaneously, as shown in Fig. 8(c). In this situation, $\tau_{2}$ will be transmitted earlier than $\tau_{5}$, which causes $\tau_{5}$ to experience the WCRT 6. However, when FQ-stations are implemented, $\tau_{5}$ arrives earlier thus it will not delayed by the transmission of $\tau_{2}$, as shown in Fig. 8(d). Therefore $R_{5}$ is decreased to 4 .

\subsection{Specific Situations where FQ-station is Better}

In a PQ-station, low-priority messages can be delayed by high-priority messages without concerning the queuing order, which ensures the transmission of high priority message as soon as possible. In contrast, in a FQ-station the priority inversion causes the high-priority messages to experience longer WCRTs, whereas it prevents the low-priority messages from being delayed by the successive high-priority messages. Hence the WCRTs of the low-priority messages will be decreased. Because a lowpriority message usually has longer WCRT than a high-priority message no matter what kind of stations are implemented, the maximum WCRT in a FQ-station is usually smaller than that in a PQ-station.

However, the WCRR is determined by the WCRT and the period. In fact, a high-priority message usually has a shorter period than a low-priority message, and the difference in the period is much larger than the difference in the WCRT. These factors cause that the maximum WCRR usually exists in the high- priority messages even if the low-priority messages have larger WCRTs. However, it also indicates that when the low-priority messages and high-priority messages have same period, implementing FQ-stations is possible to achieve higher schedulability than PQ-stations.

To verify the observations, two message sets, in which the messages of each station have the same period, are generated and the comparison experiments are conducted. The results are shown in Fig. 9. This time the implementation of FQ-stations achieves higher schedulability - the maximum WCRR is decreased when FQ-stations are implemented, and the difference of the maximum WCRR is more obvious in the message set with high bus utilization.

\section{Scheduling Messages with Both Priority and FIFO Queues}

Since both of the queues have their pros and cons in the schedulability performance, implementing the two types of queues in one CAN station is considered as a solution to obtain both the advantages. In this section, we explain our investigation on scheduling CAN messages with both priority and FIFO queues. First, in Section 6.1 we will introduce the CAN stations that are equipped with both a priority queue and a FIFO queue (called P\&F-stations). Second, in Section 6.2 we propose a messages scheduling method for the system that implements P\&F-stations. Finally, the evaluations for the implementation of P\&F-stations are shown in Section 6.3. 


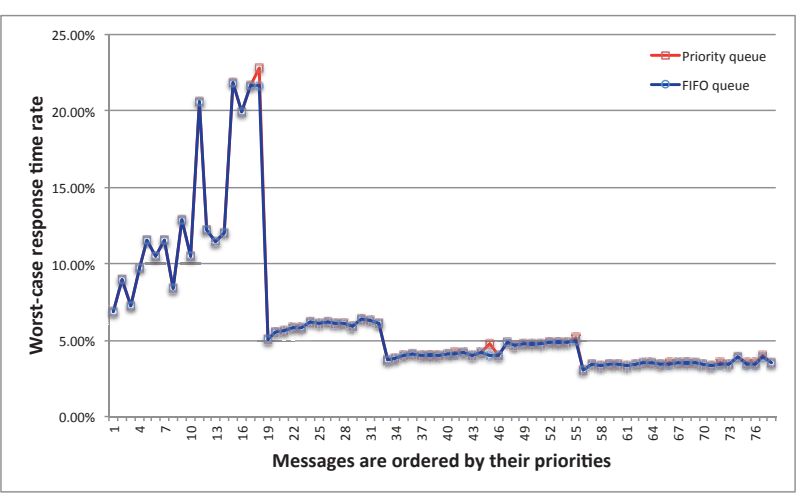

(a) The results of the message set with bus utilization $52.5 \%$

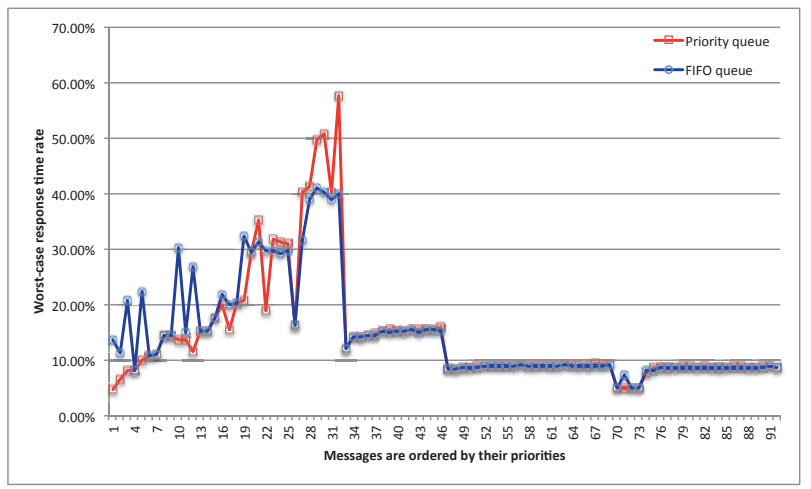

(b) The results of the message set with $79.7 \%$ bus utilization

Fig. 9 The WCRR results of the specific message sets

\subsection{P\&F-station}

A P\&F-station is designed to be equipped with both a priority queue and a FIFO queue (e.g., the R32C/160 group ECU from Renesas $\left.{ }^{(14)}\right)$. The queues can be used to queue and transmit any CAN messages in the station. When the station starts to transmit and more than one message exists in each queue, the highest priority message in the priority queue arbitrates with the oldest message in the FIFO queue. Then the higher priority message between them will be transmitted to the bus first.

In Fig. 10, we give an example to explain the transmission mechanism of a P\&F-station. In this example, the station has 4 messages, in which ID:1 and ID:2 messages are in the FIFO queue; ID:3 and ID:4 messages are in the priority queue. When the CAN controller is able to start the transmission, the oldest message (ID:2) of the FIFO queue arbitrates with the highest priority message of the priority queue (ID:3). Then the ID:2 message is selected by the CAN controller and attempts to be transmitted to CAN bus.

\subsection{The Proposed P\&F Scheduling Method}

According to the special transmission mechanism of the P\&F-station, the priority queue is able to be used to prevent the high-priority messages from being delayed by the transmission of the low-priority messages. Meanwhile, the FIFO queue can be used to decrease the maximum WCRR of the messages in the station. Our proposed messages scheduling method for P\&Fstations implemented system is described as follows:

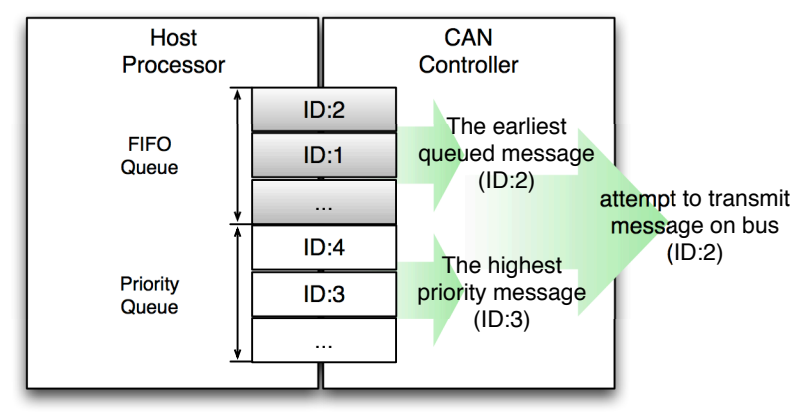

Fig. 10 The transmission mechanism of a P\&F-station

(1) Assume all the messages to be in the priority queue.

(2) Calculate the WCRRs of all messages.

(3) For each station $U_{I}$, find the $\tau_{i}\left(\tau_{i} \in U_{I}\right)$ with the maximum WCRR.

(4) Put the messages of $U_{I}$ that have same period with $\tau_{i}$ into the FIFO queue. The other messages are put into the priority queue.

\subsection{Evaluations for the Proposed Scheduling Method}

To evaluate the effectiveness of the proposed scheduling method, we conduct it on a real-world message set provided by an automobile manufacturer, and compare the schedulability among the 3 situations that all the stations are implemented as PQstations, FQ-stations and P\&F-stations, respectively. The message set has 65 messages and the bus utilization is $53.3 \%$. The automobile manufacturer has configured all the properties of the message set.

The results are shown in Fig. 11. The comparison between PQ-stations and FQ-stations, shown in Fig. 11(a), illustrates that the implementation of PQ-stations is better than FQ-stations for the message set because the maximum WCRR in PQ-stations is smaller. While when P\&F-stations are implemented and the messages are scheduled by the proposed method, the comparison between PQ-stations and P\&F-stations shows that the implementation of $\mathrm{P} \& \mathrm{~F}$-stations achieves higher schedulability, as shown in Fig. 11(b).

\section{Conclusions}

In this paper, we investigated whether and how much priority queue is better than FIFO queue when the offset assignment for CAN messages is conducted. Our results showed that for common message sets implementing the priority queues is better than the FIFO queues with respect to the schedulability. In the extreme situation, implementing the priority queues makes the maximum WCRR to be decreased $2 / 3$, comparing with the implementation of the FIFO queues.

However, we also observed that the WCRRs of most messages did not become very worse when FQ-stations are used. Moreover, implementing the FIFO queues is better in some specific situations, in which the low-priority messages and high priority-messages have same period. Considering that using the two types of queues in one station is able to obtain the both 


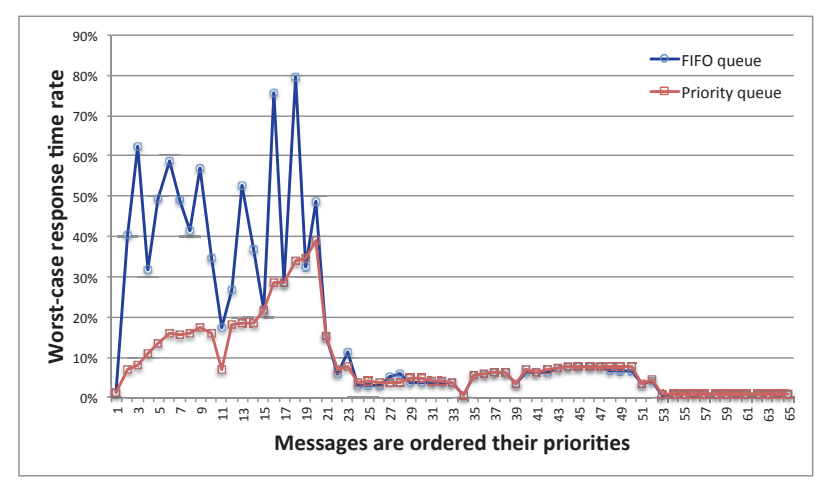

(a) The WCRR comparison between PQ-stations and FQ-stations

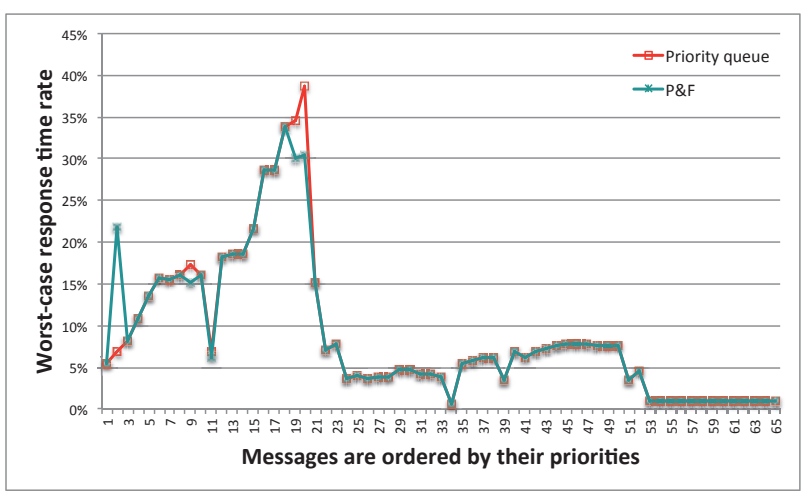

(b) The WCRR comparison between PQ-stations and P\&F-stations

Fig. 11 The WCRR comparison among PQ-stations, FQ-stations and $\mathrm{P} \& \mathrm{~F}$-stations

advantages, we proposed a new scheduling method for messages in the P\&F-station that is equipped with both a priority queue and a FIFO queue. The evaluations showed the effectiveness of the proposed scheduling method on improving the schedulability.

In the evaluating experiments of P\&F-stations, the offset assignment of the tested message set is conducted for the PQstations implemented system essentially. Therefore, a new offset assignment method for the P\&F-stations implemented system should achieve higher schedulability. This will be considered as future work.

\section{References}

(1) International Organization for Standardization, Road vehicles: Controller area network (CAN). Part 1: Data link layer and physical signaling, ISO IS11898-1 (2003).

(2) K.W. Tindell, H. Hansson, and A.J. Wellings.: Analysing Real-time communications: Controller Area Network (CAN), in Proc. 15th IEEE Real-Time Systems Symposium (RTSS'94). pp. 259-263. IEEE Computer Society Press, 1994.

(3) K.W. Tindell, A. Burns, and A.J. Wellings.: Calculating Controller Area Network (CAN) message response times (1995). Control Engineering Practice, 3(8): 1163-1169, August 1995.

(4) M. Di Natale.: Understanding and using the Controller Area network.http://inst.eecs.berkeley.edu/ ee249/fa08/Lectures/ handout_canbus2.pdf.

(5) R.I. Davis, S. Kollmann, V. Pollex, F. Slomka.: Controller Area Network (CAN) Schedulability Analysis with FIFO queues.
Proceedings of the 23rd Euromicro Conference on Real-Time Systems, IEEE, July 2011.

(6) G. Matsutani, S. Iiyama, H. Tomiyama, H. Takada.: Message Scheduling Method for CAN. Vol.2004, No.33, pp.81-86 (2004).

(7) M. Grenier, L. Havet, and N. Navet.: Scheduling frames with offsets in automotive systems: a major performance boost, in Automotive Embedded Systems Handbook (N. Navet and F. Simonot-Lion, Eds.). CRC Press/Taylor and Francis (2008).

(8) Y. Chen, R. Kurachi, G. Zeng, H. Takada.: An Offset Assignment Method for Messages of Controller Area Network. IPSJ Journal, Vol.52, No.7 pp.2245-2255, July 2011. (In Japanese)

(9) S. Iiyama, H. Tomiyama, H. Takada, M. Kido, and I. Hosotani.: Response time analysis for grouped CAN messages with offsets, IPSJ Journal, Computing System, Vol. 45, pp. 455464 (2004) (in Japanese).

(10) R. Kurachi, Y. Chen, H. Takada.: Exact Schedulability Analysis for CAN Messages with Offsets. IPSJ Journal, Vol.52, No.8, pp. 24312440, August 2011. (In Japanese)

(11) R.I. Davis, A. Burns, R.J. Bril, and J.J. Lukkien.: Controller area network (CAN) schedulability analysis: Refuted, revisited and revised. in Proc. Real-time Systems, Vol. 35, Issue 3, pp. 239272 (April 2007), ISSN:0922-6443.

(12) Y. Chen, R. Kurachi, G. Zeng, H. Takada.: The Worst-Case Response Time Analysis for FIFO-based Offset Assigned CAN Messages. IPSJ Journal, Vol.20, No.2, pp.1-12, April 2012

(13) C. Braun, L. Havet, and N. Navet.: NETCARBENCH: A benchmark for techniques and tools used in the design of automotive communication systems, in Proc. of the 7th IFAC International Conference on Fieldbuses and Networks in Industrial and Embedded Systems (FeT ' 007) (November 2007). Software and manual available at http://www.loria.fr/navet/ netcarbench/

(14) $\mathrm{R} 32 \mathrm{C} / 160$ group of $\mathrm{R} 32 \mathrm{C} / 100$ Series microcontrollers of Renesas. Documents available at: http://www.renesas.eu/ products/mpumcu/m16c/r32c100/documentation.jsp 